\title{
DPOC na população sob vigilância pela Rede Médicos Sentinela de 2007 a 2009
}

Dânia Ferreira, ${ }^{1}$ Alexandra Pina, ${ }^{2}$ Ana Margarida Cruz, ${ }^{3}$ Ana Raquel Figueiredo, ${ }^{4}$ Clara Pinto Ferreira, ${ }^{5}$ Joana Melo Cabrita, ${ }^{6}$ Jaime Correia de Sousa ${ }^{7}$

\section{RESUMO}

Objetivos: Quantificar as consultas relacionadas com DPOC dos utentes inscritos em Médicos Sentinela (MS) entre 2007 e 2009, avaliar os principais motivos de consulta relacionadas com DPOC e estimar a incidência da doença nessa população e caraterizar a terapêutica utilizada na DPOC pelos MS.

Tipo de estudo: Coorte dinâmica.

Local: Multicêntrico.

População: Utentes com idade igual ou superior a 45 anos inscritos nas listas dos MS de 2007 a 2009.

Métodos: Análise das consultas relacionadas com DPOC durante o período referido quanto à frequência e motivos de consulta e ao tratamento farmacológico instituído. Cálculo da taxa de incidência e extrapolação para a população portuguesa. Utilização dos testes qui-quadrado (comparação de proporções) e t-student (comparação de médias).

Resultados: No conjunto dos três anos, a população sob observação na Rede Sentinela foi de 106.953 indivíduos. Foram notificadas 2.916 consultas relacionadas com DPOC (62,5\% no sexo masculino), e 173 novos casos com idade igual ou superior a 45 anos ( $59,5 \%$ no sexo masculino), com média de idades de 66,9 anos, sem diferença significativa relativamente à idade entre os sexos.

Calculou-se uma taxa de incidência média anual de 161,8/100.000 (IC 95\%: 139,4-187,7/100.000), superior no sexo masculino. Os principais motivos de consulta notificados foram: renovação de medicação (61,9\%), consultas de seguimento (22,9\%) e exacerbação de sintomas (15,6\%). Foi prescrita medicação em $87,3 \%$ das consultas, com predomínio de fármacos broncodilatadores de uso inalatório: anticolinérgicos $(25,1 \%)$ e $\beta$-adrenérgicos $(20,3 \%)$.

Conclusões: A frequência de consultas e taxa de incidência foram maiores no sexo masculino, estando o valor obtido desta última aquém do esperado, presumivelmente pelo subdiagnóstico da doença e pela exclusão dos doentes que não frequentam as consultas dos CSP. O padrão de prescrição foi concordante com as recomendações da Global Initiative for Chronic Obstructive Lung Disease.

Palavras-chave: Doença Pulmonar Obstrutiva Crónica; Incidência; Vigilância Sentinela; Epidemiologia.

'Médica Interna de Ginecologia-Obstetrícia no Centro Hospitalar do Alto Ave - Guimarães; Escola de Ciências da Saúde da Universidade do Minho

${ }^{2}$ Médica Interna de Medicina Geral e Familiar na Unidade de Saúde Familiar Horizonte, Unidade Local de Saúde de Matosinhos

${ }^{3}$ Médica de Família, Unidade de Saúde Familiar Bom Porto, Agrupamento de Centros de Saúde Porto Ocidental

${ }^{4}$ Médica Interna de Medicina Geral e Familiar na Unidade de Saúde Familiar Horizonte, Unidade Local de Saúde de Matosinhos

${ }^{5}$ Médica Interna de Medicina Geral e Familiar na Unidade de Saúde Familiar Horizonte, Unidade Local de Saúde de Matosinhos

${ }^{6}$ Médica de Família na Unidade de Saúde Familiar Caravela, Unidade Local de Saúde de Matosinhos

${ }^{7}$ Professor Auxiliar Convidado, Instituto de Ciências da Vida e da Saúde (ICVS), Escola de Ciências da Saúde, Universidade do Minho e ICVS/3B 's Laboratório Associado; Médico de Família, Unidade de Saúde Familiar Horizonte, Unidade Local de Saúde de Matosinhos

\section{INTRODUÇÃO}

Doença Pulmonar Obstrutiva Crónica
(DPOC) é uma das principais causas de mor-
bilidade crónica, perda de qualidade de vida
e de mortalidade a nível mundial, associando-se a uma enorme sobrecarga a nível socioeconómico e gastos em saúde. ${ }^{1}$ Em 2004, atingia cerca de 63,6 milhões de pessoas, ${ }^{2}$ prevendo-se que, em 2030, ocupe o $3 .^{\circ}$ lugar como principal causa de morte (precedida pela doença cardíaca isquémica e doença cerebrovascular), atendendo ao provável aumento do consumo de tabaco e exposição ao fumo de combustíveis sólidos 
em ambientes fechados. ${ }^{3}$ Atingirá, assim, o sétimo lugar em termos de peso de doença. ${ }^{4}$

A prevalência de DPOC oscila entre 4 e $20 \%$ nos adultos com mais de 40 anos, aumentando com a idade, sobretudo entre os fumadores. ${ }^{5}$ Rondará os 4 a $10 \%$ na população adulta europeia, ${ }^{6}$ e estudos que reportam a realidade portuguesa apontam para taxas de prevalência de $5,3 \%$ nos homens e $4,0 \%$ nas mulheres. ${ }^{7}$

A incidência estimada em Portugal aponta para valores de 3,1 por 1000 nos homens e 1,7 por 1000 nas mulheres, que aumentam a partir dos 40 anos. ${ }^{7} \mathrm{Um}$ estudo mais recente, efetuado na população da grande Lisboa com idades iguais ou superiores a 40 anos, ${ }^{8}$ mostrou uma prevalência de DPOC de 14,2\%, em estádios iguais e superiores ao 1 da Classificação GOLD (The Global Initiative for Chronic Obstructive Lung Disease), ${ }^{9}$ o que vem confirmar a ideia generalizada de que a doença se encontra em crescimento e está, de facto, subdiagnosticada. ${ }^{10}$

Em Portugal, a DPOC constitui a segunda causa de internamento por doença respiratória, associando-se a elevada frequência de consultas médicas e de serviços de urgência, assim como por um significativo número de internamentos hospitalares. ${ }^{10}$ Acarreta ainda custos relacionados com o consumo de fármacos e de oxigenoterapia e ventiloterapia domiciliárias de longa duração. ${ }^{1}$

A caracterização epidemiológica da situação da DPOC em Portugal permitirá construir estratégias para minimizar os impactos negativos da doença na sociedade, através da prevenção e tratamento desta doença respiratória. ${ }^{11} \mathrm{O}$ envolvimento dos médicos de família na gestão de grande parte dos doentes com DPOC torna pertinente a investigação da sua prevalência, assim como a carga de trabalho gerada pelo acompanhamento destes doentes crónicos. ${ }^{10}$

No sentido de melhorar o conhecimento epidemiológico desta patologia, a Rede Médicos-Sentinela (MS) procedeu, durante o triénio 2007 a 2009, ao registo e notificação de consultas relacionadas com DPOC ocorridas na população de utentes que mantém sob vigilância, visando caracterizar a população que consulta o seu médico de família por motivos relacionados com esta doença. ${ }^{12,13}$

Assim, os objetivos deste trabalho foram:

1. Quantificar as consultas relacionadas com DPOC nos utentes das listas de médicos pertencentes à Rede Médicos-Sentinela (MS) no triénio 20072009;

2. Avaliar os principais motivos de consulta relacionados com DPOC nessa população durante o período do estudo;

3. Estimar as taxas de incidência anuais da DPOC, bem como o número de novos casos da doença para a população portuguesa durante o triénio 2007-2009;

4. Caracterizar a terapêutica utilizada para DPOC nesta população.

\section{MÉTODOS}

Foi realizado um estudo de coorte dinâmica das consultas (presenciais e não presenciais) relacionadas com DPOC ocorridas nas listas dos Médicos Sentinela durante o triénio 2007-2009..$^{14,15}$

A Rede «Médicos-Sentinela» (MS) é um sistema de observação em saúde, constituído por médicos de família, cuja atividade profissional é desenvolvida em Centros de Saúde. Esta rede tem como principais objetivos estimar taxas de incidência de algumas doenças ou de situações com elas relacionadas que ocorrem na população inscrita, fazer a vigilância epidemiológica de algumas doenças que ocorrem na comunidade, de forma a permitir a identificação precoce de eventuais «surtos», e constituir uma base de dados que possibilite, em qualquer momento, a análise epidemiológica aprofundada de doenças com interesse para a saúde pública.

O presente estudo incluiu todas as consultas realizadas pelos Médicos-Sentinela notificadores entre 2007 e 2009, a indivíduos com idade igual ou superior a 45 anos e com DPOC. Durante os três anos do estudo não é seguida efetivamente sempre a mesma população, sendo possível que tenham entrado ou saído médicos da rede e tenha havido modificações nas listas em análise. Para além disso, não foram excluídos do denominador os utentes com diagnóstico já conhecido de DPOC.

As variáveis estudadas foram: idade e sexo do utente, motivo da consulta relacionada com DPOC (início de sintomas, alteração do padrão de sintomas, exacerbação, renovação de medicação, consulta de seguimento e/ou outros), tipo de diagnóstico da doença 
(caso novo, caso conhecido ou caso sem diagnóstico), atitude médica na consulta (aconselhamento/esclarecimento do utente, referenciação e/ou prescrição de medicação) e medicação prescrita relacionada com a patologia em estudo.

Com relação ao diagnóstico de DPOC, os casos notificados foram classificados como: "Casos novos», onde se incluíram os novos diagnósticos de DPOC notificados nos três anos de estudo, «Casos conhecidos", que incluíram todos os casos de DPOC com diagnóstico prévio conhecido pelo médico, e em «Casos sem diagnóstico», que incluiu todos as situações ainda em estudo e também consultas relacionadas com pedidos de esclarecimento, aconselhamento, atestados e declarações a pessoas sem diagnóstico. O conhecimento do médico face ao diagnóstico era mutuamente exclusivo, sendo que na descrição da medicação podia ser incluído mais do que um fármaco. ${ }^{16}$

Com base nos dados obtidos procedeu-se ao cálculo da frequência de consultas, que corresponde ao número de consultas relacionadas com DPOC gerado por cada mil utentes com idade igual ou superior a 45 anos inscritos nas listas dos médicos sentinela notificadores, permitindo avaliar a carga de trabalho associada à doença na consulta de medicina geral e familiar (MGF). Foram também analisadas as consultas relacionadas com «novos casos» de DPOC, de maneira a estimar a taxa de incidência anual da doença.

Para proceder à extrapolação dos resultados para a população portuguesa teve-se em conta a população média com idade acima dos 45 anos em dezembro de $2008 .{ }^{17}$

Os cálculos efetuados utilizaram apenas a população com idade igual ou superior a 45 anos em função da frequência de notificações ter sido reduzida abaixo dessa idade e pelo facto de DPOC se desenvolver nos indivíduos expostos sobretudo a partir das quarta e quinta décadas de vida (acima dos 35-40 anos). ${ }^{18,19}$

A colheita dos dados foi realizada utilizando o boletim de notificação criado pela Rede MS, em papel ou em formato eletrónico, que é preenchido pelo médico e enviado semanalmente para o Observatório Nacional de Saúde, onde os dados são registados numa base de dados informática.

A análise estatística descritiva e analítica da base de dados criada foi feita com os programas SPSS $®$ (Statis- tical Package for Social Sciences) versão 17.0 e Epi In$\mathrm{fo}^{\mathrm{TM}}$, recorrendo-se aos testes qui-quadrado para a comparação de proporções e $t$-student para a comparação de médias. O nível de significância adotado foi de 0,05 .

O presente trabalho foi elaborado no contexto de uma rede de investigação no âmbito do Ministério da Saúde e utilizou dados recolhidos de forma anónima pelos médicos de família da Rede Médicos Sentinela.

\section{RESULTADOS}

\section{População sob observação efetiva}

Durante o triénio 2007-2009, a população sob observação efetiva acima dos 45 anos, definida pelo somatório do total de utentes inscritos nas listas dos MS em cada ano do período em estudo, foi de 106.953 indivíduos, sendo $55,0 \%$ do sexo feminino. A este valor correspondeu uma população média anual de 35.651.

\section{Consultas relacionadas com DPOC de 2007 a 2009}

Durante este período, o número de consultas relacionadas com DPOC foi de 2.916, com uma média de idades de 72,1 anos, mínimo de 45 e máximo de 97 anos, desvio padrão de 10,4. A maioria das consultas foi realizada a utentes do sexo masculino $(62,5 \%)$ com uma média de idades de 72,0 anos e de 72,3 anos nos utentes do sexo feminino, não se verificando diferenças com significado estatístico entre os dois valores $(p=0,365)$.

\section{Frequência de consultas relacionadas com DPOC por 1000 inscritos}

A frequência de consultas foi de 27,3/1000 inscritos: $37,9 / 1000$ para o sexo masculino e 18,6/1000 para o sexo feminino, aumentando progressivamente com a idade e atingindo o valor máximo de 58,1/1000 no grupo etário dos 75 ou mais anos. Verificou-se também que houve um predomínio de consultas a utentes do sexo masculino com o aumento da idade. Para todos os grupos, à exceção do «45-54 anos», verificaram-se diferenças estatisticamente significativas na frequência de consultas entre os sexos ( $p<0,001)$ (Quadro I).

\section{Conhecimento do médico face ao diagnóstico}

Nas consultas relacionadas com DPOC, $91,8 \%$ dos casos já eram conhecidos, 6,2\% constituíram novos diagnósticos e em 0,9\% não havia ainda um diagnóstico es- 


\begin{tabular}{|c|c|c|c|c|}
\hline \multicolumn{5}{|c|}{$\begin{array}{l}\text { QUADRO I. Frequência de consultas relacionadas com DPOC, por } \\
\text { sexo e grupo etário, por cada } 1000 \text { inscritos, de } 2007 \text { a } 2009 .\end{array}$} \\
\hline $\begin{array}{l}\text { Grupo Etário } \\
\text { (anos) }\end{array}$ & $\begin{array}{l}\text { Masculino } \\
\mathrm{n}(\%)\end{array}$ & $\begin{array}{l}\text { Feminino } \\
\mathrm{n}(\%)\end{array}$ & $\begin{array}{l}\text { Total } \\
\mathrm{n}(\%)\end{array}$ & $p$ \\
\hline $45-54$ & $75(4,7)$ & $106(6,2)$ & $181(5,5)$ & 0,07 \\
\hline $55-64$ & $376(29,0)$ & $181(12,4)$ & $557(20,2)$ & $<0,001$ \\
\hline $65-74$ & $575(54,4)$ & $304(22,6)$ & $879(36,6)$ & $<0,001$ \\
\hline$\geq 75$ & $796(91,7)$ & $503(36,8)$ & $1299(58,1)$ & $<0,001$ \\
\hline Total & $1822(37,9)$ & $1094(18,6)$ & $2916(27,3)$ & $<0,001$ \\
\hline
\end{tabular}

Teste qui-quadrado

tabelecido. Em 1,1\% das situações a resposta não foi assinalada por parte do médico notificador.

\section{Motivos de consulta relacionada com DPOC}

Não sendo mutuamente exclusivos, os motivos mais representados foram renovação de medicação $(62,0 \%)$ e consultas de seguimento (32,0\%), como se pode observar no Quadro II.

\section{Tratamento farmacológico prescrito}

Ao longo deste período foi efetuada prescrição em $89,6 \%$ das consultas. Esta consistiu maioritariamente em fármacos broncodilatadores de uso inalatório, nomeadamente anticolinérgicos $(25,1 \%)$ e $\beta$-adrenérgi$\cos (20,3 \%)$. No Quadro III listam-se os fármacos prescritos de acordo com a classificação Anatomical Therapeutic Chemical da Organização Mundial de Saúde. ${ }^{20}$

Das 952 prescrições de agonistas $\beta 2$ seletivos, usados isoladamente e por via inalatória, verifica-se que 55,6\% $(n=529)$ corresponderam a agonistas de longa duração de ação. Relativamente aos anticolinérgicos, os de longa duração corresponderam a 69,9\% (n=826).

\section{Novos casos de DPOC de 2007 a 2009}

No período em estudo foram notificados 173 novos casos de DPOC, com uma média de idade de 66,9 anos, mínimo de 45 e máximo de 90 anos, desvio padrão de 10,4 . A maioria dos novos casos ocorreu no sexo masculino (59,5\%). A média de idades foi de 66,0 para o sexo masculino e de 68,4 anos para o sexo feminino, não havendo diferenças estatisticamente significativas entre as médias $(\mathrm{p}=0,137)$.

\section{Taxa de incidência média anual de DPOC de 2007 a 2009}

A taxa de incidência média anual de DPOC estimada para ambos os sexos, no período de 2007 a 2009, foi de 161,8/ 100.000 (IC 95\%: 139,4187,7), sendo de 214,2/100.000 (IC 95\%: 176,7$259,7)$ para o sexo masculino e $118,9 / 100.000$ (IC 95\%: 94,2-150,0) para o sexo feminino (Quadro IV). As taxas de incidência de DPOC foram significativamente maiores no sexo masculino, no total ( $\mathrm{p}<0,001)$ e nos grupos etários 55-64 e 65-74 anos ( $p=0,015$ e 0,004 respetivamente).

\section{Evolução da taxa de incidência de DPOC ano a ano}

Ao longo do período em estudo, a taxa de incidência anual de DPOC atingiu o valor mínimo de 141,1/100.000 (IC 95\%: 103,4-192,8) em 2008 e máximo de 190,5/100.000 (IC 95\%: 148,7-244,1) em 2009 (Quadro V). Não se verificaram diferenças estatisticamente significativas entre os valores de cada ano.

\section{Ajustamento à população portuguesa, de 2007 a 2009:}

Extrapolando a taxa de 161,8 casos/ 100.000 para a estimativa da população residente em Portugal em 2008 com idade igual ou superior a 45 anos (4.593.652 indivíduos), ${ }^{17}$ a média estimada de novos diagnósticos é, assim, de 7.432 novos casos de DPOC por ano em todo o país (Quadro VI).

\begin{tabular}{|c|c|c|}
\hline \multicolumn{3}{|c|}{$\begin{array}{l}\text { QUADRO II. Distribuição das consultas relacionadas } \\
\text { com DPOC, segundo o motivo de consulta. }\end{array}$} \\
\hline Tipo de consulta & $\mathrm{n}$ & $\%$ \\
\hline Início de sintomas & 96 & 3,3 \\
\hline Alteração do padrão de sintomas & 142 & 4,9 \\
\hline Exacerbação & 456 & 15,6 \\
\hline Renovação de medicação & 1809 & 62,0 \\
\hline Consulta de seguimento & 934 & 32,0 \\
\hline Outros & 130 & 4,5 \\
\hline
\end{tabular}




\begin{tabular}{|c|c|c|c|c|c|c|}
\hline \multicolumn{4}{|c|}{ Fármacos } & \multicolumn{2}{|c|}{ N. ${ }^{\circ}$ de Prescrições } & $\%(n=4700)$ \\
\hline \multicolumn{4}{|l|}{ Oxigénio } & & 293 & 6,2 \\
\hline \multirow{9}{*}{$\begin{array}{l}\text { Fármacos para } \\
\text { doença } \\
\text { obstrutiva das } \\
\text { vias aéreas }\end{array}$} & \multirow{3}{*}{$\begin{array}{l}\text { Adrenérgicos para } \\
\text { inalação }\end{array}$} & \multirow{2}{*}{$\beta$-agonistas } & Longa ação* & 529 & \multirow{2}{*}{952} & \multirow{2}{*}{20,3} \\
\hline & & & Curta ação* & 423 & & \\
\hline & & \multicolumn{2}{|c|}{$\begin{array}{l}\text { Adrenérgicos e outros medicamentos } \\
\text { (associações com agonistas } \beta \text { ) }\end{array}$} & \multicolumn{2}{|r|}{713} & 15,2 \\
\hline & \multirow{3}{*}{$\begin{array}{l}\text { Outros fármacos } \\
\text { inalados }\end{array}$} & \multicolumn{2}{|l|}{ Glucocorticoides } & \multicolumn{2}{|r|}{464} & 9,9 \\
\hline & & \multirow{2}{*}{ Anticolinérgicos } & Longa ação* & 826 & \multirow{2}{*}{1181} & \multirow{2}{*}{25,1} \\
\hline & & & Curta ação* & 355 & & \\
\hline & $\begin{array}{l}\text { Adrenérgicos para uso } \\
\text { sistémico }\end{array}$ & \multicolumn{2}{|c|}{$\begin{array}{l}\text { Agonistas seletivos dos } \\
\text { adrenoceptores beta }\end{array}$} & \multicolumn{2}{|r|}{54} & 1,2 \\
\hline & Outros fármacos de uso & \multicolumn{2}{|l|}{ Xantinas } & & 515 & 10,9 \\
\hline & sistémico & \multicolumn{2}{|c|}{ Antagonistas do recetor leucotrieno } & & 143 & 3,0 \\
\hline $\begin{array}{l}\text { Preparados } \\
\text { contra a tosse e } \\
\text { resfriados }\end{array}$ & $\begin{array}{l}\text { Expetorantes, excluindo } \\
\text { associação com } \\
\text { antitússicos }\end{array}$ & \multicolumn{2}{|l|}{ Mucolíticos } & & 210 & 4,5 \\
\hline \multicolumn{4}{|c|}{ Anti-histamínicos para uso sistémico } & & 19 & 0,4 \\
\hline \multicolumn{4}{|c|}{ Preparados de ação na orofaringe } & & 4 & 0,1 \\
\hline \multirow{3}{*}{ Vacinas } & \multirow{3}{*}{\multicolumn{3}{|c|}{$\begin{array}{l}\text { Vacina antipneumocócica } \\
\text { Outras vacinas bacterianas } \\
\text { Vacina sazonal da gripe }\end{array}$}} & & 25 & 0,5 \\
\hline & & & & & 4 & 0,1 \\
\hline & & & & & 52 & 1,1 \\
\hline \multicolumn{4}{|c|}{ Corticosteroides de uso sistémico } & & 71 & 1,5 \\
\hline
\end{tabular}

* Esta subdivisão não existe na classificação ATC.

\section{DISCUSSÃO}

Neste estudo de três anos e considerando os utentes com idade igual ou superior a 45 anos, obteve-se uma população sob observação efetiva (PSOE) anual média de 35.651 utentes, representando 106.953 utentes em observação nos três anos. Durante este período, foram notificados 173 novos casos de DPOC, o que leva a uma taxa de incidência de 161,8 casos / 100.000 , que é superior nos homens $(214,2$ casos/100.000).

Relativamente à frequência de consultas, verificou-se um predomínio de consultas a utentes do sexo masculino e a frequência de consultas aumentou progressivamente com a idade, e atingindo o valor máximo de $58,1 / 1000$ no grupo etário dos 75 ou mais anos.
A Rede Médicos Sentinela é constituída por médicos que participam de forma voluntária, o que possibilita uma elevada notificação de casos, uma boa qualidade geral da informação e a caracterização de forma detalhada da atividade clínica relacionada com DPOC neste grupo.

Reconhecem-se limitações associadas à utilização de dados provenientes da notificação dos médicos da Rede MS. Podem decorrer erros de notificação por imprecisões no diagnóstico, uma vez que não são definidos critérios diagnóstico nem se pergunta ao médico qual o método utilizado para a confirmação do mesmo, por duplicação de notificações e por alterações na notificação em períodos de inatividade do médico. Estas 


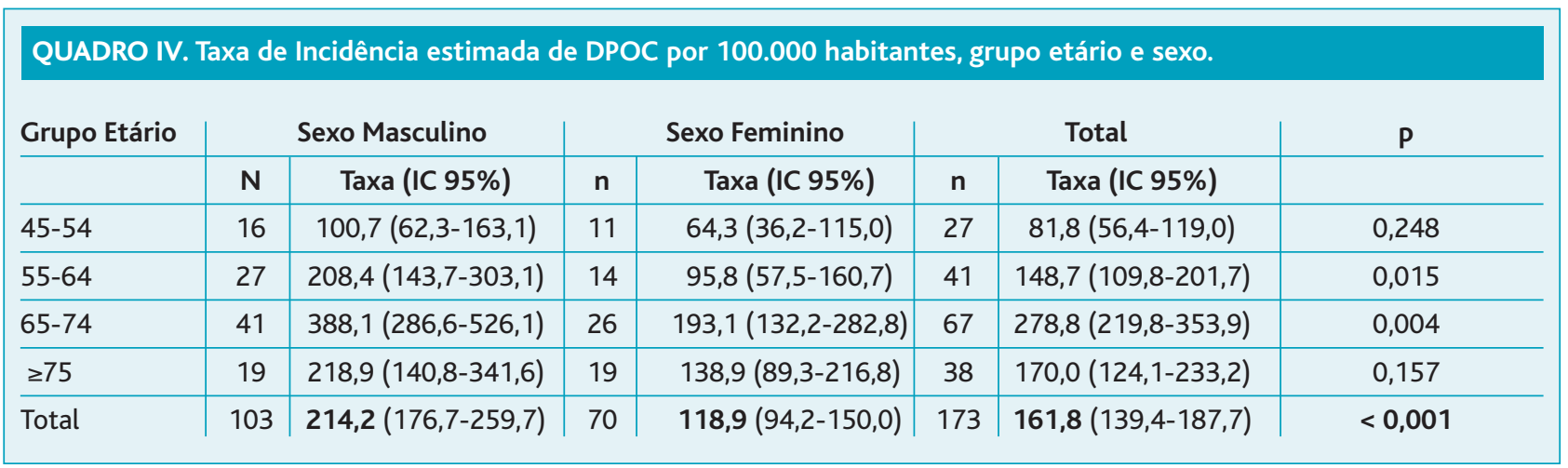

Teste qui-quadrado IC 95\% - intervalo de confiança a 95\%.

\section{QUADRO V. Taxa de incidência estimada em cada ano.}

\begin{tabular}{l|c|c|c} 
Ano & $\mathbf{n}$ & $\begin{array}{c}\text { População sob } \\
\text { observação efetiva }\end{array}$ & $\begin{array}{c}\text { Taxa incidência estimada } \\
\text { por } 10^{5} \text { (IC 95\%) }\end{array}$ \\
\hline 2007 & 72 & 46.759 & $154,0(122,4-193,9)$ \\
\hline 2008 & 39 & 27.642 & $141,1(103,4-192,8)$ \\
\hline 2009 & 62 & 32.552 & $190,5(148,7-244,1)$ \\
\hline Média & 173 & 106.953 & $161,8(139,4-187,7)$ \\
\hline
\end{tabular}

IC $95 \%$ - intervalo de confiança a $95 \%$.

situações podem levar quer a sub quer a sobrenotificação de casos.

Ao tratar-se de uma amostra de conveniência de médicos de família, a PSOE pode não ser representativa da população portuguesa, uma vez que não foi selecionada de forma aleatória. Alguns grupos profissionais, que dispõem de subsistemas de saúde, e os estratos sociais mais elevados ficam provavelmente sub-representados.
Por outro lado, as variações das listas dos médicos (por transferências ou óbitos) e o facto de existirem utentes não utilizadores (que não estão na realidade em observação) faz com que a população sob observação tenha tendência para ser progressivamente sobrestimada e as taxas de incidência subestimadas.

Estudos realizados na Rede MS mostraram também que os indivíduos com 75 e mais anos estão ligeiramente sobrerepresentados na amostra de PSOE e que a PSOE não é representativa da população portuguesa em termos de distribuição geográfica.

A frequência de consultas encontrada foi de 27,3/1000. Um estudo do Reino Unido decorrido entre 1991 e 1992 aponta para taxas de consulta de 41,7/1000 habitantes dos 45-64 anos, 88,6/1000 dos 6574 anos e 103,2/1000 dos 75 aos 84 anos. $^{6}$

De acordo com o European Lung White Book e relativamente ao número médio de consultas médicas por doenças respiratórias major em alguns países europeus, as consultas por DPOC ultrapassam em larga escala as consultas por asma, pneumonia, neoplasia maligna da traqueia e pulmões e tuberculose. ${ }^{6}$

\section{QUADRO VI. Estimativas de novos casos de DPOC ocorridos na população portuguesa de 2007 a 2009, ajustado à população portuguesa.}

População residente em Portugal com idade $\geq 45$ anos

\begin{tabular}{l|c|c|c|c|c|c|c|c}
\multicolumn{2}{c}{ com idade $\geq 45$ anos } & \multicolumn{2}{c}{ Taxa de incidência por $10^{5}$ (IC 95\%) } & \multicolumn{3}{c}{ na população portuguesa } \\
\hline Homens & Mulheres & Total & Homens & Mulheres & Total & Homens & Mulheres & Total \\
\hline 2.089 .649 & 2.504 .003 & 4.593 .652 & $\begin{array}{c}214,2 \\
(176,7-259,7)\end{array}$ & $\begin{array}{c}118,9 \\
(94,2-150,0)\end{array}$ & $\begin{array}{c}161,8 \\
(139,4-187,7)\end{array}$ & 4.476 & 2.977 & 7.432
\end{tabular}

Estimativa de novos casos ocorridos na população portuguesa 
As diferenças entre sexos foram concordantes com outros trabalhos, onde se verificou maior carga de trabalho com indivíduos do sexo masculino. Um estudo escocês publicado em 2010 encontrou taxas de consulta para DPOC ou insuficiência cardíaca menores em mulheres e em grupos etários extremos (idade inferior a 55 ou superior ou igual a 85 anos). ${ }^{21}$ Outro estudo, também escocês, encontrou taxas de consulta nos cuidados de saúde primários por insuficiência cardíaca de $17,2 / 1000$ para os dois sexos, sendo superior na mulher $(17,9 / 1000)$, que aumentaram progressivamente com a idade. ${ }^{22}$ No entanto, tanto no global como isoladamente, os valores encontrados nesse estudo apontam para uma carga de trabalho menor com insuficiência cardíaca do que com DPOC. Em contrapartida, e relativamente aos indivíduos do sexo masculino e comparando com os nossos resultados, este estudo escocês aponta para uma carga de trabalho relacionada com hipertensão arterial de 96,2/ 1000, valor bastante superior ao por nós encontrado para DPOC (37,9/1000).

Estes resultados refletem a carga de trabalho de uma doença de declínio progressivo de função, sendo de esperar que o volume de trabalho gerado pela doença aumente com a idade.

Os motivos de consulta mais representados são expectáveis tendo em conta que se trata de uma doença crónica de evolução progressiva, que exige terapêutica de controlo/alívio, com a respetiva carga de consultas de seguimento associada.

Quanto ao tratamento farmacológico da DPOC, no total de prescrições efetuadas pelos MS notificadores verificou-se um predomínio dos fármacos broncodilatadores de uso inalatório, nomeadamente anticolinérgicos $(25,1 \%)$ e $\beta$-adrenérgicos (20,3\%), logo seguidos pelas associações de fármacos com $\beta$-agonistas $(15,2 \%)$. Apesar de ainda nenhum tratamento farmacológico ser indiscutivelmente capaz de travar o declínio da função pulmonar que caracteriza a evolução da DPOC, os broncodilatadores constituem fármacos centrais no controlo sintomático da doença e na redução das exacerbações, concretamente os agonistas $\beta 2$-adrenérgicos, anticolinérgicos e metilxantinas, usados isoladamente ou em combinação. ${ }^{9}$

Já a opção dos MS por agonistas adrenérgicos de uso sistémico (cerca de 1,2\%) não foi tão significativa, o que condiz com as recomendações internacionais, que de- fendem que $\beta 2$ - agonistas administrados por via oral têm uma ação mais lenta e implicam mais efeitos laterais do que quando usados por via inalatória. ${ }^{23}$

Considerando os broncodilatadores inalatórios prescritos, o maior volume de prescrições correspondeu a fármacos de longa duração de ação, o que também respeita as normas orientadoras da GOLD, que definem o uso regular destes medicamentos como mais efetivo e conveniente do que a terapia com broncodilatadores de curta duração de ação. ${ }^{24}$

A prescrição de xantinas, correspondente a cerca de $11 \%$ do total de prescrições, não foi, neste estudo, tão significativa quanto a dos outros broncodilatadores, tendência também defendida nas diretrizes da GOLD, que privilegiam o uso de broncodilatadores inalatórios em detrimento da teofilina sistémica, pelo seu maior risco de toxicidade. ${ }^{9}$

Cerca de 9,9\% do total de prescrições medicamentosas foram de corticosteroides inalatórios, valor que na realidade está subestimado, uma vez que muitas das associações $\operatorname{com} \beta$ - agonistas poderão incluir corticosteroide na associação. Apesar de não modificarem o declínio a longo prazo do $\mathrm{FEV}_{1}$, o tratamento regular com corticosteroides inalados reduz a frequência das exacerbações em pacientes sintomáticos com DPOC em estádios 3 e 4 e com exacerbações repetidas da doença, ${ }^{25}$ benefícios que parecem ampliar-se com a sua associação a um $\beta 2$-agonista de longa duração de ação. ${ }^{26}$ Por outro lado, a evidência científica atualmente disponível não é suficiente para recomendar o tratamento com cursos prolongados de corticosteroide sistémico, que neste estudo respondem apenas por 1,5\% do total de prescrições, sendo estes fármacos apenas efetivos no tratamento das exacerbações da doença. ${ }^{9}$

Quanto à prescrição de oxigénio, um dos principais tratamentos não farmacológicos para doentes com DPOC em estádio IV, ${ }^{27}$ correspondeu a $6,2 \%$ do total de prescrições efetuadas pelos MS. Este valor pode estar subestimado relativamente à percentagem real, já que os doentes com insuficiência respiratória crónica, principais consumidores da oxigenoterapia domiciliária de longa duração, poderão ter consultado um pneumologista responsável pelo seu acompanhamento e vigilância hospitalares em consulta externa da especialidade e, portanto, não renovar a sua medicação nos Cuidados de Saúde Primários. 
O uso regular e generalizado de agentes mucolíticos, que neste estudo representam apenas cerca de $4,5 \%$ do total de prescrições, não é atualmente recomendado na DPOC, embora haja alguma evidência científica que suporta o benefício destes fármacos na redução de exacerbações em doentes não tratados com corticosteroides inalados. ${ }^{28}$

Cerca de 3\% das prescrições corresponderam a antagonistas dos leucotrienos, fármacos que na realidade não estão recomendados no tratamento farmacológico da DPOC, mas sim como adjuvantes da corticoterapia inalada no controlo da asma. ${ }^{29}$ Estes resultados podem estar relacionados com erros de registo de medicação não relacionada com DPOC em doentes que tenham simultaneamente DPOC e asma.

A vacinação antigripal é recomendada nos doentes com DPOC na medida em que pode reduzir a incidência de doença grave e morte em cerca de $50 \% .{ }^{30} \mathrm{~A}$ vacina antipneumocócica também está recomendada em doentes com DPOC de idade igual e superior a 65 anos, mostrando reduzir a incidência de pneumonia adquirida na comunidade em pacientes com idade inferior a 65 anos e FEV1 inferior a 40\%. ${ }^{31}$ No entanto, a percentagem de prescrições das vacinas antigripal e antipneumocócica ficou aquém do esperado, tendo em conta a população de utentes consumidores da consulta relacionada com DPOC (média de idades de 72 anos).

Esta subprescrição pode ser devida a vários fatores, entre os quais o facto de muitos médicos não entenderem a vacinação como medicação relacionada com DPOC (tema debatido na apresentação dos resultados na reunião anual da rede), o desconhecimento dos médicos para a necessidade de vacinar estes doentes, a recusa do doente em ser vacinado e ausência de registos por parte do médico prescritor.

Relativamente à vacina antipneumocócica, é importante realçar que a frequência de administração é menor que a da vacina antigripal, pelo que a taxa encontrada pode não ser assim tão baixa. Muitos doentes poderão ter já realizado a vacinação antes do período de tempo em análise, atendendo à média de idades encontrada.

À semelhança de outros estudos da Rede Médicos-Sentinela, o cálculo da taxa de incidência tem limitações relacionadas com a população em estudo, dado que ao utilizar-se a PSOE como denominador da fórmula estamos a incluir utentes que já têm o diagnóstico de DPOC e como tal não estão em risco de desenvolver a doença. Assim, apenas poderemos obter taxas de incidência estimadas, considerando que a PSOE estará toda ela em risco de desenvolver DPOC, subestimando os valores reais. Este facto dificulta a comparação com outros estudos que avaliaram a incidência de DPOC.

A taxa de incidência anual estimada de DPOC foi de 161,8/100.000. O estudo de van Durme que estudou indivíduos com idade igual ou superior a 55 anos, durante um período médio de 11 anos, obteve uma taxa de incidência global de 920/100.000 pessoas/ano (IC 95\%: 850-1000). ${ }^{32}$ De Marco e colaboradores obtiveram uma taxa anual de incidência de 280/ 100.000 (IC 95\%: 230-330), numa população de adultos com idades compreendidas entre os 20 e os 44 anos, num estudo coorte com a duração de 10 anos. ${ }^{33}$

No estudo de Geijer e colegas a taxa de incidência cumulativa de DPOC moderada (estádio II da GOLD) a cinco anos, em homens com idades compreendidas entre os 40 e os 65 anos, foi de 8,3\% (IC 95\%: 5,8-11,4). ${ }^{34}$

Um estudo norueguês de Johannessen et al, que incluiu indivíduos dos 18 aos 74 anos, encontrou uma taxa de incidência cumulativa de $6,1 \%$ a 9 anos (IC $95 \%$ : $4,0-8,1)$, o que corresponde a uma taxa de incidência anual média de $0,7 \%$ na população estudada. ${ }^{35}$

$\mathrm{O}$ uso de diferentes definições de DPOC, de valores de função pulmonar sem broncodilatação e as diferenças na inclusão de determinados estádios de DPOC torna difícil a comparação dos valores estimados entre estudos e com os deste estudo, que se baseia na notificação dos casos, sem que fique explícita a forma de se chegar ao diagnóstico.

Verificou-se um aumento não estatisticamente significativo nas taxas de incidência estimadas entre 2008 e 2009. Uma razão para estes resultados pode ser um efeito conhecido na rede, relacionado com um aumento do número de notificações nos anos seguintes à apresentação dos resultados na reunião anual da rede dos médicos-sentinela.

A extrapolação da estimativa da taxa de incidência obtida, 161,8 por 100.000 , para uma população residente em Portugal em 2008 com idade igual ou superior a 45 anos estimada em 4.593.652 indivíduos, per- 
mitiu calcular uma média estimada de novos diagnósticos de 7.432 novos casos de DPOC por ano, em todo o país. ${ }^{17}$ Este cálculo apresenta algumas limitações em função da representatividade da amostra já discutida anteriormente e das limitações no rigor dos cálculos da população residente, estimada pelo Instituto Nacional de Estatística, mas carecendo da confirmação que virá a ser possível quando estiverem disponíveis os dados do Censo de 2011.

Apesar das limitações já referidas, com este estudo foi possível obter-se uma estimativa da incidência de DPOC em Portugal. Estudos futuros com métodos diferentes, poderão contribuir para calcular com maior exatidão uma taxa de incidência de DPOC, determinar se esta é, em Portugal, diferente da observada noutros países, estudar incidências específicas em determinados grupos de risco e avaliar o peso desta doença nas consultas de medicina geral e familiar.

\section{Artigo escrito ao abrigo do novo acordo ortográfico.}

\section{AGRADECIMENTOS}

Os autores agradecem a todos os médicos da Rede Médicos Sentinela e à coordenação da Rede toda a colaboração prestada na recolha e fornecimento dos dados.

\section{REFERÊNCIAS BIBLIOGRÁFICAS}

1. Direção-Geral da Saúde. Programa Nacional de Prevenção e Controlo da Doença Pulmonar Obstrutiva Crónica. Lisboa: Direção-Geral da Saúde, Divisão de Doenças Genéticas, Crónicas e Geriátricas; 2005.

2. World Health Organization. The Global Burden of Disease: 2004 Update. Geneva:World Health Organization; 2008.

3. World Health Organization. World Health Statistics 2008. Geneva: World Health Organization; 2008.

4. Mathers $C D$, Loncar D. Projections of global mortality and burden of disease from 2002 to 2030. PLoS Med 2006 Nov; 3 (11): e442.

5. Direção-Geral da Saúde. Vigilância global, prevenção e controlo das doenças respiratórias crónicas - uma abordagem integradora. Lisboa: Direção-Geral da Saúde; 2007.

6. European Respiratory Society. The European Lung White Book. Brussels: European Respiratory Society / European Lung Foundation; 2003.

7. Borges M, Gouveia M, Costa J, Pinheiro LS, Paulo S, Carneiro AV. Carga da doença atribuível ao tabagismo em Portugal. Rev Port Pneumol 2009 Nov-Dec; 15 (6): 951-1004.

8. Bárbara C, Rodrigues F, Dias H, Cardoso J, Almeida J, Matos MJ, et al. COPD prevalence in Portugal. The Burden of Obstructive Lung Disease study (BOLD). Congresso Anual da European Respiratory Society, Barcelona, 18 a 22 de setembro de 2010. Disponível em: https://www.ersnetsecure.org/public/prg_congres.detail?ww_i_session $=17164 \& w w \_c$ _mode=TEXTE-SPECIAL [acedido em 31.05.2011].
9. Global Initiative for Chronic Obstructive Lung Disease. Global Strategy for Diagnosis, Management, and Prevention of COPD. Updated 2010. Disponível em: http://www.goldcopd.com [acedido em 15.05.2011].

10. Araújo AT. Relatório do Observatório Nacional das Doenças Respiratórias 2009. Disponível em http://www.ondr.org/relatorios_ondr.html [acedido em 31/05/2011].

11. Fundação Portuguesa do Pulmão. Documento de Consenso da Fundação Portuguesa do Pulmão: proposta para a elaboração dum Plano Nacional de Prevenção e Controle das Doenças Respiratórias. Lisboa: Fundação Portuguesa do Pulmão; 2010.

12. Instituto Nacional de Saúde Dr. Ricardo Jorge. Denominadores: quem são? como se calculam? Disponível em: http://www.insa.pt/sites/INSA/ Portugues/AreasCientificas/Epidemiologia/Unidades/UnInstrObser/Do cuments/proj_ms_denominadores_onsa.pdf [acedido em 29/05/2011].

13. Rede Médicos-Sentinela. O que se fez em 2007. Lisboa: Departamento de Epidemiologia do Instituto Nacional de Saúde Dr. Ricardo Jorge; 2009.

14. Noordzij M, Dekker FW, Zoccali C, Jager KJ. Measures of disease frequency: prevalence and incidence. Nephron Clin Pract 2010; 115 (1): c17-20.

15. Hoogendoorn M, Rutten-van Mölken MP, Hoogenveen RT, van Genugten ML, Buist AS, Wouters EF, et al. A dynamic population model of disease progression in COPD. Eur Respir J 2005 Aug; 26 (2): 223-33.

16. Médicos-Sentinela. O que se fez em 2008. Departamento de Epidemiologia. Lisboa: Instituto Nacional de Saúde Dr. Ricardo Jorge; 2010. p. 109-110. Disponível em: http://www.insa.pt/sites/INSA/Portugues/Publicacoes/Outros/Documents/Epidemiologia/Relatorio_MS_22 _2008.pdf [acedido em 29/05/2011].

17. Instituto Nacional de Estatística. Estimativas provisórias de população residente, 2008 Portugal, NUTS II, NUTS III e municípios. Lisboa: Instituto Nacional de Estatística; 2009.

18. Stratelis G, Jakobsson P, Molstad S, Zetterstrom O. Early detection of COPD in primary care: screening by invitation of smokers aged 40 to 55 years. Br J Gen Pract 2004 Mar; 54 (500): 201-6.

19. Ulrik CS, Løkke A, Dahl R, Dollerup J, Hansen G, Cording PH, et al; TOP study group. Early detection of COPD in general practice. Int J Chron Obstruct Pulmon Dis 2011; 6: 123-7.

20. WHO Collaborating Centre for Drug Statistics Methodology. Anatomictherapeutic-chemical classification of drugs (ATC) Classification index. Oslo: Norwegian Institute of Public Health; 2005. Disponível em: http://www.whocc.no/atc_ddd_index/ [acedido em 09/12/2009].

21. Hawkins NM, Jhund PS, Simpson CR, Petrie MC, Macdonald MR, Dunn FG, et al. Primary care burden and treatment of patients with heart failure and chronic obstructive pulmonary disease in Scotland. Eur J Heart Fail 2010 Jan; 12 (1): 17-24.

22. Murphy NF, Simpson CR, McAlister FA, Stewart S, Maclntyre K, Kirkpatrick $M$, et al. National survey of the prevalence, incidence, primary care burden, and treatment of heart failure in Scotland. Heart 2004 Oct; 90 (10): 1129-36.

23. Shim CS, Williams MH Jr. Bronchodilator response to oral aminophylline and terbutaline versus aerosol albuterol in patients with chronic obstructive pulmonary disease. Am J Med 1983 Oct; 75 (4): 697-701.

24. Oostenbrink JB, Rutten-van Mölken MP, Al MJ, Van Noord JA, Vincken 
W. One-year cost-effectiveness of tiotropium versus ipratropium to treat chronic obstructive pulmonary disease. Eur Respir J 2004 Feb; 23 (2): 241-9.

25. Calverley P, Pauwels R, Vestbo J, Jones P, Pride N, Gulsvik A, et al. Combined salmeterol and fluticasone in the treatment of chronic obstructive pulmonary disease: a randomised controlled trial. Lancet $2003 \mathrm{Feb}$ 8; 361 (9356): 449-56.

26. Calverley PM, Boonsawat W, Cseke Z, Zhong N, Peterson S, Olsson H. Maintenance therapy with budesonide and formoterol in chronic obstructive pulmonary disease. Eur Respir J 2003 Dec; 22 (6): 912-9.

27. Siafakas NM, Vermeire P, Pride NB, Paoletti P, Gibson J, Howard P, et al. Optimal assessment and management of chronic obstructive pulmonary disease (COPD). The European Respiratory Society Task Force. Eur Respir J 1995 Aug; 8 (8): 1250-1.

28. Zheng JP, Kang J, Huang SG, Chen P, Yao WZ, Yang L, et al. Effect of carbocisteine on acute exacerbation of chronic obstructive pulmonary disease (PEACE Study): a randomised placebo-controlled study. Lancet 2008 Jun 14; 371 (9629): 2013-8.

29. Global Initiative for Asthma (2010). Global Strategy for Asthma Management and Prevention (Updated 2010). Disponível em: http://guideline.gov/content.aspx?id=32556 [acedido em 29/05/2011]

30. Wongsurakiat P, Maranetra KN, Wasi C, Kositanont U, Dejsomritrutai $\mathrm{W}$, Charoenratanakul S.Acute respiratory illness in patients with COPD and the effectiveness of influenza vaccination: a randomized controlled study. Chest 2004 Jun; 125 (6): 2011-20.

31. Alfageme I, Vazquez R, Reyes N, Muñoz J, Fernández A, Hernandez M, et al. Clinical efficacy of antipneumococcal vaccination in patients with COPD. Thorax 2006 Mar; 61 (3): 189-95.

32. Van Durme YM, Verhamme KM, Stijnen T, van Rooij FJ, Van Pottelberge GR, et al. Prevalence, incidence, and lifetime risk for the develop- ment of COPD in the elderly: the Rotterdam study. Chest 2009 Feb; 135 (2): 368-77.

33. De Marco R, Accordini S, Cerveri I, Corsico A, Antó JM, Künzli N, et al. Incidence of chronic obstructive pulmonary disease in a cohort of young adults according to the presence of chronic cough and phlegm. Am J Respir Crit Care Med 2007 Jan; 175 (1): 32-9.

34. Geijer RM, Sachs AP, Verheij TJ, Salomé PL, Lammers JW, Hoes AW. Incidence and determinants of moderate COPD (COLD II) in male smokers aged 40-65 years: 5-year follow up. Br J Gen Pract 2006 Sep; 56 (530): 656-61.

35. Johannessen A, Omenaas E, Bakke P, Gulsvik A. Incidence of GOLD-defined chronic obstructive pulmonary disease in a general adult population. Int J Tuberc Lung Dis 2005 Aug; 9 (8): 926-32.

\section{CONFLITOS DE INTERESSE}

Jaime Correia de Sousa foi membro não remunerado da Fundação AstraZeneca Portugal e recebeu esporadicamente honorários como consultor ou palestrante da Altana (Nycomed), MSD e Novartis. Os restantes autores declaram não possuir qualquer tipo de conflitos de interesses.

\section{FINANCIAMENTO}

Este estudo não recebeu qualquer financiamento externo.

\section{ENDEREÇO PARA CORRESPONDÊNCIA}

Dânia Isabel Costa Ferreira

Rua S. Domingos, N. 164 , 2. ${ }^{\circ}$ Dto., 4710-435 Braga

E-mail: daniaisabelferreira@gmail.com

Recebido em 12/02/2012

Aceite para publicação em 12/07/2012 


\section{ABSTRACT \\ COPD IN THE POPULATION UNDER SURVEILLANCE BY THE PORTUGUESE SENTINEL PRACTICE NETWORK FROM 2007 TO 2009}

Objectives: To determine the number of chronic obstructive pulmonary disease (COPD)-related consultations among patients registered in the Portuguese Sentinel Practice Network (PSPN) between 2007 and 2009, to calculate the reasons for visits related to COPD, to estimate the incidence of COPD, and to characterize COPD-related therapy.

Study design: Dynamic cohort.

Setting: Muticentric.

Participants: Patients $\geq 45$ years old from the lists of general practitioners in the PSPN between 2007 and 2009.

Methods: A study of COPD-related consultations over a three-year period, reporting the frequency, the reasons for consultation and treatments was conducted. The estimated incidence rate of COPD was calculated and extrapolated to the Portuguese population. The chi-square test to compare proportions and the t-student test for comparison of means were used.

Results: During the study period, the population under observation was 106,953 individuals and 2,916 consultations related to COPD were reported. Of these, $62.5 \%$ were for males. New cases of COPD were reported in 173 persons $\geq 45$ years old, of which $59.5 \%$ were males, with a median age of 66.9 years,. No significant age difference was found between genders. We calculated an annual incidence rate of 161.8/100,000 (95\% Cl: 139.4-187.7), higher in men. The reasons for consulting were renewal of prescriptions (61.9\%), follow-up appointments (22.9\%) and exacerbation of symptoms (15.6\%). Medication was prescribed in $87.3 \%$ of consultations, mainly for inhaled bronchodilators, including anti-cholinergic drugs (25.1\%) and $\beta$ adrenergic agonists $(20.3 \%)$.

Conclusions: There were more COPD-related visits and a higher incidence rate of COPD for men, though the value observed was lower than expected. This was probably due to under-diagnosis or to the fact that patients who do not attend Primary Health Care clinics were excluded. The prescription pattern found was consistent with the recommendations of the Global Initiative for Chronic Obstructive Lung Disease.

Keywords: Chronic Obstructive Pulmonary Disease; Incidence; Sentinel Surveillance; Epidemiology. 